\title{
Foxq1 promotes metastasis of nasopharyngeal carcinoma by inducing vasculogenic mimicry via the EGFR signaling pathway
}

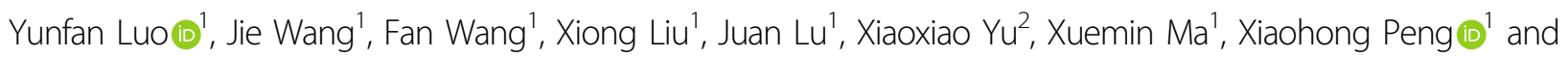
Xiangping Li ${ }^{1}$

\begin{abstract}
In nasopharyngeal carcinoma (NPC), the treatment of tumor metastasis and recurrence is challenging and is associated with poor clinical efficacy. Vasculogenic mimicry (VM) is a new blood-supply model of malignant tumor that is closely related to tumors' distant metastasis. Our previous study demonstrated that miR-124 could target Foxq1 to inhibit NPC metastasis. Whether Foxq1 affects metastasis through vasculogenic mimicry is worth consideration. In this study, we show that VM formation positively correlates with the expression of Foxq1, and EGFR, and the TNM stage in 114 NPC patient samples. Meanwhile, we show that VM-positive NPC patients have a poor prognosis. Furthermore, using in vitro and vivo approaches, we confirm that Foxq1 has a significant effect on NPC metastasis through promoting VM formation, which could be effectively inhibited by EGFR inhibitors (Nimotuzumab or Erlotinib). Also a synergistic efficacy of anti-EGFR and anti-VEGF drugs has been found in NPC inhibition. Mechanistically, the luciferase reporter gene and CHIP assays show that Foxq1 directly binds to the EGFR promoter region and regulates EGFR transcription. In conclusion, our results show that Foxq1 is regulated by miR-124 and that it promotes NPC metastasis by inducing VM via the EGFR signaling pathway. Overall, these results provide a new theoretical support and a novel target selection for anti-VM therapy in the treatment of nasopharyngeal carcinoma.
\end{abstract}

\section{Introduction}

Nasopharyngeal carcinoma is a malignant tumor that has a high incidence in Southeast Asia, especially in southern China. Owing to the significant improvements in our understanding of the pathogenesis of nasopharyngeal carcinoma and the progress that has been made in diagnosis and treatment technologies, the survival rate of nasopharyngeal carcinoma has been significantly improved ${ }^{1,2}$. However, recurrence or distant metastasis is still the biggest challenge in the treatment of nasopharyngeal carcinoma, and the main cause of patients' death ${ }^{3}$. Hence, clarifying the

\footnotetext{
Correspondence: Xiaohong Peng (418897077@qq.com) or

Xiangping Li (li321162@qq.com)

'Otorhinolaryngology Head and Neck Surgery, Nanfang Hospital of Southern Medical University, Guangzhou, China

2Otorhinolaryngology Head and Neck Surgery, Guangzhou Women and Children's Medical Center, Guangzhou, China

Edited by N. Barlev
}

metastatic mechanism of NPC is key in improving the survival rate of NPC patients.

Angiogenesis is an essential process of malignant tumors' growth and metastasis ${ }^{4}$. For a long time, angiogenesis was believed to be the only blood-supply model for tumors; Thus, anti-angiogenic therapy was considered as an effective treatment for preventing metastasis. Currently, anti-angiogenic drugs mainly target the VEGF/ VEGFR signaling pathway, however, anti-VEGF drugs have not achieved certain clinical effects in the treatment of nasopharyngeal carcinoma ${ }^{5,6}$. Therefore, it is particularly important to investigate the causes that are associated with the poor efficacy of anti-angiogenic drugs in nasopharyngeal carcinoma.

Vasculogenic mimicry (VM) is a novel blood-supply system that was first proposed in invasive human melanoma by Maniotis et al. in $1999^{7}$. In 2016, the journal

\section{(c) The Author(s) 2021}

(c) (i) Open Access This article is licensed under a Creative Commons Attribution 4.0 International License, which permits use, sharing, adaptation, distribution and reproduction c. in any medium or format, as long as you give appropriate credit to the original author(s) and the source, provide a link to the Creative Commons license, and indicate if changes were made. The images or other third party material in this article are included in the article's Creative Commons license, unless indicated otherwise in a credit line to the material. If material is not included in the article's Creative Commons license and your intended use is not permitted by statutory regulation or exceeds the permitted use, you will need to obtain permission directly from the copyright holder. To view a copy of this license, visit http://creativecommons.org/licenses/by/4.0/. 
Science noted that vasculogenic mimicry supplement traditional angiogenesis in supplying nutrients to tumors ${ }^{8}$. The discovery of VM, which is a vascular network pattern formed by highly invasive tumor cells that replace endothelial cells, partly explained the resistance to antiangiogenic drugs ${ }^{9,10}$. Previous reports have also preliminarily confirmed that single anti-angiogenic therapy were ineffective in inhibiting VM, which might impair the effectiveness of anti-angiogenic approaches ${ }^{11-13}$. Recently, increasing evidences indicated that VM exists in numerous solid tumors, including head and neck carcinoma, and breast, liver, ovarian, gastric, and prostate cancers ${ }^{14}$, and it played an important role in tumor metastasis ${ }^{15}$. In nasopharyngeal carcinoma, there were only few studies on vasculogenic mimicry ${ }^{16,17}$, while neither of these focused on VM role in NPC metastasis. VM-specific regulatory mechanism in nasopharyngeal carcinoma remains to be further explored, which may lead to the discovery of new therapeutic targets.

In a previous study, we have demonstrated that miR-124 could target Foxq1 to inhibit NPC metastasis ${ }^{18}$. However, the specific mechanism of NPC metastasis that was induced by Foxq1, remained elusive. Foxq1 is a transcription factor that belongs to the Fox family, and is one of the key regulators of multiple cellular functions and plays an important role in tumors' development, invasion, and metastasis ${ }^{19}$. Recently, studies on Foxq1 oncogenic and metastatic roles, have received extensive attentions ${ }^{20,21}$. Although the relationship between Foxq1 and VM has not been reported, other studies have shown that Foxq1 promotes tumor metastasis by promoting epithelial-mesenchymal transition (EMT) in a variety of tumors, including gastric, lung, colorectal, breast, and bladder cancers ${ }^{22-24}$. Meanwhile, EMT was closely related to VM formation, and EMT-related transcription factors, such as Twist, Snail and ZEB1, were highly upregulated in VM forming tumor cells ${ }^{25-27}$. These findings demonstrated that EMT-related transcription factors play a significant role in VM formation. Therefore, whether foxq1 affects the metastasis of nasopharyngeal carcinoma by regulating VM is worthy of further study.

In this study, we found that the epidermal growth factor receptor (EGFR) is directly regulated by Foxq1. EGFR high expression has been shown to correlate with poor prognosis in NPC patients with undifferentiated nasopharyngeal carcinoma ${ }^{28}$. Unlike anti-angiogenic therapy, the combination of anti-EGFR drugs, such as Nimotuzumab, and Cetuximab, with chemoradiotherapy have been proved to maximize the survival of stage II-IVb NPC patients $^{29}$. However, the exact mechanism of anti-EGFR therapy in the treatment of NPC is still unclear. Meanwhile, as EGFR has been reported as an important regulator of VM in various malignancies ${ }^{30,31}$, we considered that VM may be the main leading cause that is associated with the difference in efficacy of anti-angiogenic drugs and anti-EGFR drugs in nasopharyngeal carcinoma. Thus in this manuscript, we examined the effect of anti-EGFR drugs on VM formation and the combined effect of antiEGFR and anti-angiogenic drugs in NPC.

In summary, our study aimed at clarifying whether Foxq1 promotes NPC metastasis through regulating VM formation and confirm the specific functional relationship between Foxq1 and EGFR in promoting VM formation. We also provide a novel theoretical support for anti-EGFR therapy and clarify the possible causes that are related to the poor efficacy of anti-VEGF drugs in NPC.

\section{Materials and methods \\ Clinical samples}

The NPC tissue specimens $(n=114)$ were obtained from the Nanfang Hospital of Southern Medical University (GuangZhou, China). They were collected by biopsy from NPC patients between 2007 and 2019, and pathologically confirmed as NPC. Detailed pathological, clinical data and survival durations of the NPC patients were collected for all samples through outpatient and telephone follow-ups. The control tissue specimens $(n=$ 40) were collected from biopsy specimen of nasopharyngitis. Informed written consent was obtained from each patient. The TNM classification was performed according to the definitions of the seventh edition of the UICCAmerican Joint Committee on Cancer staging criteria. The use of these tissue samples was approved by the Ethics Committee of Southern Hospital of Southern Medical University.

\section{Immunohistochemical and CD31-PAS dual staining}

Formalin-fixed, and paraffin-embedded tissues were sectioned at $4 \mathrm{~mm}$ thickness, then harvested and fixed in $4 \%$ paraformaldehyde overnight at $4{ }^{\circ} \mathrm{C}$. The antigen blocking was performed using 10\% goat serum (AR0009, Boster,China). The sections were probed overnight at $4{ }^{\circ} \mathrm{C}$ with primary anti-Foxq1 (PA5-40772, Invitrogen, USA), anti-CD31 (ab134168, Abcam, USA) and anti-EGFR (ZM0093, Zsbio, China) antibodies. The staining was detected using the DAB system (ZLI-9017, Zsbio, China). To detect VM structures, a PAS staining kit (G1281, Solarbio, China) and anti-CD31 (ab28364, Abcam, USA) were used. The numbers of positive cells were counted from $\geq 5$ randomly chosen fields and at $\times 400$ magnification, by two independent pathologists.

\section{Cell culture}

All NPC cells were acquired from the Cancer Research Center of Southern Medical University and cultured in RPMI-1640 medium (PM15101,Thermo Fisher Scientific, USA), supplemented with $10 \%$ fetal bovine serum (10270-106, Thermo Fisher Scientific, 
USA), $100 \mathrm{mg} / \mathrm{ml}$ streptomycin (15140-122, Thermo Fisher Scientific, USA), and $100 \mathrm{U} / \mathrm{ml}$ penicillin (15140122, Thermo Fisher Scientific, USA), and maintained at $37^{\circ} \mathrm{C}$ in a humidified atmosphere with $5 \% \mathrm{CO}_{2}$.

\section{RNA isolation, reverse transcription, and quantitative real- time PCR}

Total RNA was extracted from the samples using RNAiso Plus (R401-01, Vazyme, China) and reversely transcribed to cDNA using the HiScipt III RT SuperMix for Quantitative Real-time PCR (+gDNA wiper) (R32301, Vazyme, China). The quantitative reverse transcription PCR (qRT-PCR) was performed using ChamQ SYBR qRT-PCR Master Mix (Low ROX Premixed) (Q331-02, Vazyme, China) on an ABI QuantStudio5 System. GAPDH was used as the mRNAs endogenous control. All samples were normalized to the internal control, and the relative expression levels were calculated using the relative quantification assay. The primer sequences for qRTPCR are shown in Additional file 1.

\section{Western blot}

The proteins were extracted from the samples using the radio immunoprecipitation assay lysis buffer (P0013B, Beyotime, China), containing a protease-inhibitor cocktail (HY-K0010, MCE, USA). The proteins were lysed in SDSloading buffer (FD006, Fdbio, China), then the lysates were resolved on sodium dodecyl sulphate-polyacrylamide gel electrophoresis and transferred to polyvinylidene fluoride membrane (IPVH00010, Millipore, USA). The membrane was incubated with polyclonal antibodies against EGFR (AF6043, Affinity, China), phospho-EGFR (AF3047, Affinity, China), MMP2 (AF0577, Affinity, China), MMP9 (AF5228, Affinity, China), VE-Cadherin (AF6265, Affinity, China), AKT (bsm-33282M, Bioss, China), phospho-AKT (bs-0876R, Bioss, China), Foxq1 (PA1-31951, Invitrogen, USA) or GAPDH (AC033, Abclonal, China) at a dilution of $1: 1000$, then incubated with species-specific HRP-conjugated secondary antibodies at a dilution of 1:5000. The immunoreactive bands were visualized by enhanced chemiluminescence (WBKLS0100, Millipore, USA). phosphatase inhibitor cocktail (HY-K0021, MCE, USA) was used for the phosphoprotein blots, e.g., p-EGFR and p-AKT.

\section{Cell transfection}

The full-length sequences of Foxq1 were amplified by PCR and cloned into the pCMV3 vector (HG20110-UT, Sino Biological, China) to construct pCMV3-Foxq1 overexpressing plasmid. Plasmids were transfected into the cells using the Lipofectamine 3000 transfection reagent (L3000008, Thermo Fisher Scientific, USA). Lentiviruses that overexpress miR-124 or Foxq1 knockdown shorthairpin RNA (Genechem, China) were used to infect NPC cell lines and according to the manufacture's instruction.

\section{Dual-luciferase assay}

Before transfection, 293T cells $\left(1 \times 10^{5}\right.$ cells $)$ were plated in a 24-well plate for $24 \mathrm{~h}$ and co-transfected with the control pcDNA3.1 plasmid $(2.0 \mu \mathrm{g} / \mathrm{ml})$ or the Foxq1 plasmid $(2.0 \mu \mathrm{g} / \mathrm{ml})$ together with the control vector pGL3 $(2.0 \mu \mathrm{g} / \mathrm{ml})$ or the EGFR plasmid $(2.0 \mu \mathrm{g} / \mathrm{ml})$ using Lipofectamine 3000 (L3000008,Thermo Fisher Scientific, USA). After $36 \mathrm{~h}$ incubation, cell lysates were prepared and firefly/renilla luciferase values were quantified using the Dual-Luciferase Reporter Assay System (E1910, Promega, USA) on a GloMax-96 plate reader (Promega).

\section{CHIP-PCR}

CHIP experiment was performed using the EZ-Magna $\mathrm{ChIP}^{\mathrm{TM}}$ A/G (17-10086, Millipore, USA) and according to the manufacture's instruction. Anti-Foxq1 antibodies (sc166266, SantaCruz, USA) were used for immunoprecipitation and the bound DNA was detected using two primer sets that were designed against the human EGFR promoter (for the sequences see Additional file 1).

\section{Three-dimensional culture}

A 24-well plate was coated with $100 \mu$ l growth factorreduced Matrigel (354230, BD Biosciences, USA), that was polymerized at $37^{\circ} \mathrm{C}$ for $1 \mathrm{~h}$, after which $1 \times 10^{5}$ cells that were suspended in $500 \mu \mathrm{l}$ of medium containing $10 \%$ FBS, were plated on the surface of the gel and incubated at $37^{\circ} \mathrm{C}$ for $24 \mathrm{~h}$. Three wells were provided for each group. The cells were then photographed under an inverted microscope (IX71, OLYMPUS, Japan). The Statistical results of the average number of the tubular structures were counted by ImageJ.

\section{Immunofluorescence staining}

The cells were fixed with $4 \%$ paraformaldehyde for $15 \mathrm{~min}$, then blocked with $1 \% \mathrm{BSA}$ at $37^{\circ} \mathrm{C}$ for $30 \mathrm{~min}$ and incubated with an Foxq1 mouse monoclonal antibody (H00094234-M05, Abnova, USA), or a rabbit polyclonal antibody against EGFR, MMP2, MMP9 or VE-Cadherin. Then, the cells were washed with PBS and incubated at room temperature for $1 \mathrm{~h}$ with a secondary antibody conjugated with Alexa Fluor 555 (A0453, Beyotime, China). Finally, the cells were incubated with a mounting medium (with DAPI) (S2110, Solarbio, China) at room temperature for $3 \mathrm{~min}$ and all samples were observed using a scanning microscope (BX63, OLYMPUS, Japan)

\section{Tumor xenograft model and tumor metastasis assay in vivo}

Four to 5-week-old BALB/C nude mice were purchased from the Center of Laboratory Animal of Southern Medical University, and the animal protocol was approved by the Institutional Animal Care and Use Committee of Southern Medical University. The animals were grouped randomly. 


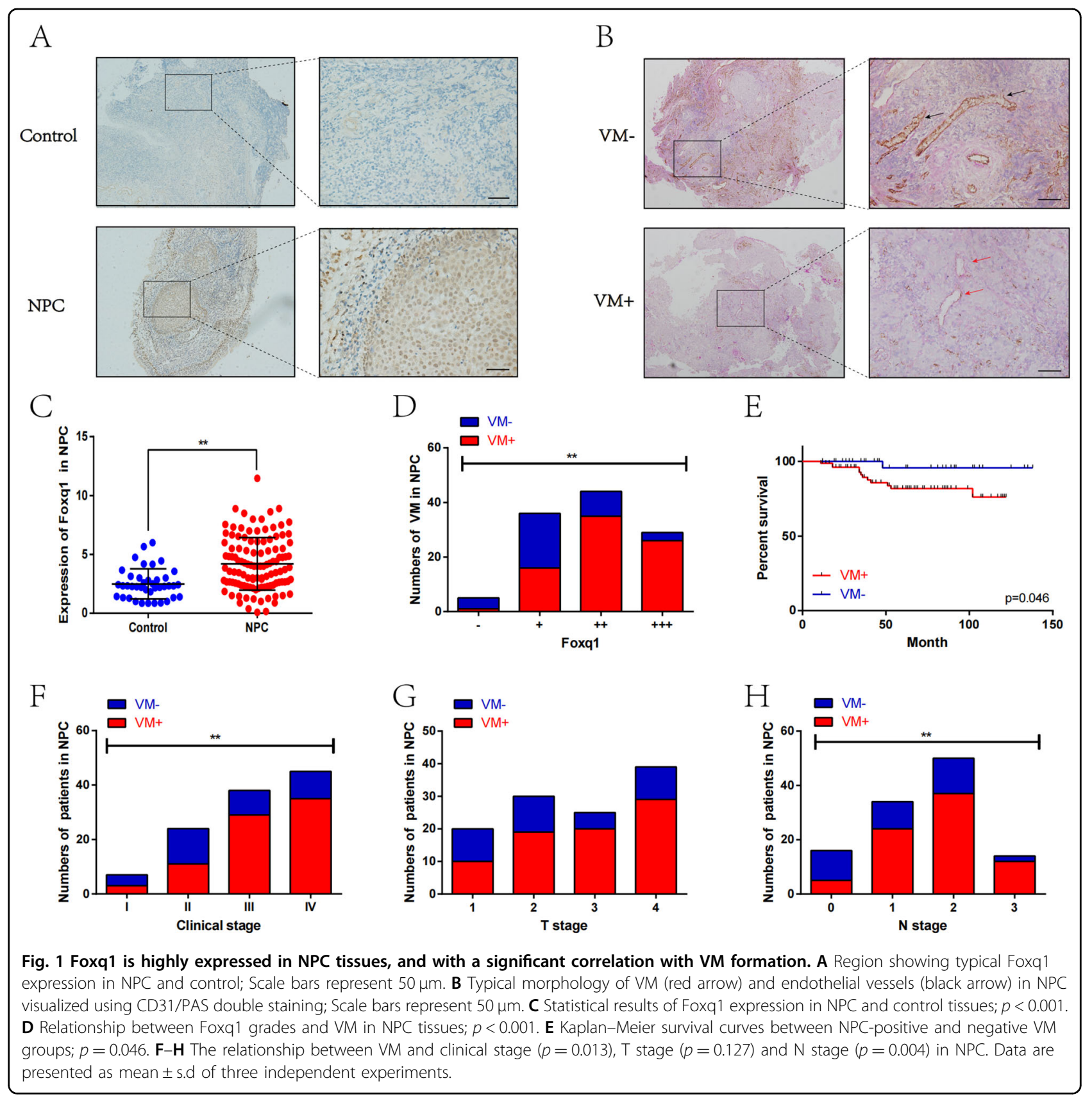

For the subcutaneous animal model, control and Foxq1 overexpressing $5-8 \mathrm{~F}$ cells, were injected subcutaneously into the flank of BALB/c nude mice, respectively $\left(1 \times 10^{6}\right.$ cells/ mouse; $n=6$ /group). The tumor volumes were monitored every 3 days. Two weeks later, the mice were euthanized, and the tumors were dissected out for further analyses. For the in vivo tumor metastatic assay, we used the lung metastatic model. Control and Foxq1 overexpressing 5-8F cells were injected through tail vein into BALB/c nude mice, respectively $\left(1 \times 10^{6}\right.$ cells/mouse; $n=6 /$ group $)$. Five weeks later, the mice were euthanized, and the lung metastatic nodules were counted. For the drug sensitivity experiment,
Foxq1 overexpressing $5-8 \mathrm{~F}$ cells were separately inoculated into the flanks or the tail vein of the BALB/C nude mice, and randomly divided into three groups $\left(1 \times 10^{6}\right.$ cells/mouse; $n$ $=6 /$ group), and treated with Erlotinib (i.g., $50 \mathrm{mg} / \mathrm{kg}$ ), Nimotuzumab (i.v., $20 \mathrm{mg} / \mathrm{kg}$ ), or physiological saline, respectively. After the mice were euthanized, the xenograft tumors were collected, and the incidences of lung metastases were recorded.

\section{Statistical analyses}

The SPSS 25.0 software was used for statistical analyses. All data were from at least three independent 
experiments. The two-tailed student's $t$-test was used for comparing two independent groups. The two-tailed Mann-Whitney test was used to analyze the data with non-Gaussian distribution. The Gaussian distribution data was analyzed by Welch's correction if variances, which was determined by the $F$-test, when significantly different. One-way ANOVA analysis of variance was used to compare multiple groups. The Kaplan-Meier method and log-rank test were used to construct survival curves. The relationship between Foxq1 and EGFR was analyzed using the Spearman's correlation analysis. The data are shown as the mean \pm SEM unless otherwise. $p$-values of $<0.05$ were considered statistically significant.

\section{Results}

Foxq1 expression is high in NPC and significantly correlates with VM formation

Using IHC staining, Foxq1 expression was quantified in control and NPC tissues, containing 114 NPC patient samples $(7,24,38$, and 45 cases of grade I, II, III, and IV) and 40 nasopharyngitis samples. The results showed that Foxq1 was significantly upregulated in NPC tissues compared with nasopharyngitis tissues $(p<0.001)$ (Fig. 1A, C and Fig. S1). Thereafter, we assessed the VM vessels (CD31-negative, PAS-positive) in 114 NPC samples. Images of the VM typical morphology (red arrow) and endothelial vessels (black arrow) are shown in Fig. 1B. The immunohistochemical staining showed that $68 \%$ of the samples (78/114) were VM-positive, and $32 \%$ of the samples (36/114) were VM-negative. The statistical analysis indicated a strong correlation between VM and Foxq1 $(p<0.001)$ (Fig. 1D). Meanwhile, VM was significantly related with clinical stage $(p=0.013)($ Fig. $1 \mathrm{~F})$ and $\mathrm{N}$ stage $(p=0.004)($ Fig. $1 \mathrm{H})$, but not with $\mathrm{T}$ stage $(p=0.127)$ (Fig. $1 \mathrm{G})$, age $(p=0.662)$ or sex $(p=0.577)$ (Table 1$)$. The result of the Kaplan-Meier analysis indicated that VMpositive NPC patients had shorter survival times than the VM-negative patients $(p=0.046)$ (Fig. 1E). Taken together, Foxq1 is highly expressed in NPC tissues, while VM, which potentially predicts poor prognosis in NPC patients, is clinically correlated with Foxq1 expression.

\section{Foxq1 promotes VM formation in vitro}

We first confirmed that NPC cell lines, including 5-8F, 6-10B, CNE1, CNE2 and C666-1, could form different degrees of vessel-like structures in 3D culture (Fig. 2A, B). It was obvious that poorly differentiated NPC cells $(5-8 \mathrm{~F}$, CNE2, and C666-1) had a greater ability to form vessellike structures compared to highly differentiated NPC cells (6-10B, CNE1), suggesting that the VM formation ability of NPC cells significantly correlates with the degree of malignancy. To investigate Foxq1 function in regulating VM formation, stably transfected NPC cell lines
(5-8F, CNE1) that were knocked down or overexpressed Foxq1 were established. The expression of Foxq1 in these cells was investigated by qRT-PCR and western blot (Fig. $2 \mathrm{C}-\mathrm{F})$. A three-dimensional cell culture system was used to characterize the VM formation capacity of the dysregulated-Foxq1 cells. As Foxq1-depleted NPC cells exhibited a loss of VM formation capacity, the Foxq1 overexpressing cells could significantly promote VM formation (Fig. 2G-J). These results imply that Foxq1 contributes to VM generation in NPC cells.

\section{Foxq1 increased the expression of EGFR and VM-related genes, while Erlotinib inhibited Foxq1-induced VM formation}

Although Foxq1 expression positively correlates with $\mathrm{VM}$, the exact mechanism of this event is still unclear. Using bioinformatics databases (Oncomine, GEO, TCGA), we selected some genes as miR-124 and Foxq1 possible targets. QRT-PCR was used to verify the expression of these genes in the $5-8 \mathrm{~F}$ cell line that overexpressed miR-124 or the down-regulated Foxq1 (Fig. 3A, B). Hence, EGFR was chosen as a Foxq1 downstream target gene. Subsequently, qRT-PCR and western blot were used to assess the expression of EGFR in the 5-8f and CNE1 cell lines that overexpressed or had a Foxq1 knockdowns. The results showed that Foxq1 overexpression significantly promotes EGFR expression, while Foxq1 downregulation inhibits EGFR expression (Fig. 3C-F). To confirm whether Foxq1 exerts a VM promoting effect through EGFR in NPC cells, the Foxq1 overexpressing NPC cells were pre-treated with Erlotinib (EGFR inhibitor) for $24 \mathrm{~h}$, then the capacity of VM formation was detected by 3D culture. As expected, Erlotinib markedly abrogated the promotion of VM formation that was induced by Foxq1 (Fig. 3G, H). The above results confirmed that Foxq1 promotes VM formation through regulating EGFR expression in vitro. In NPC tissues, the Pearson correlation analysis showed a significant correlation between Foxq1 and EGFR $\left(p<0.001, r^{2}=0.5303\right)$ (Fig. 3I, J), and that EGFR significantly correlates with VM $(p<0.001)$ (Fig. 3K).

Furthermore, qRT-PCR, western blot, and immunofluorescence staining were used to evaluate Foxq1 effect on the EGFR signaling pathway and VM-related genes. The results showed that the expression of genes, such as AKT, p-AKT, BCL-2, MMP2, MMP9, and VE-cadherin, significantly and positively correlate with Foxq1 expression (Fig. 4A-E). These results support our hypothesis that Foxq1 is involved in VM formation trough the EGFR signaling pathway in NPC.

\section{Foxq1 is a direct transcriptional regulator of EGFR}

To further explore the specific interaction between Foxq1 and EGFR, we used UCSC and JASPAR databases 
Table 1 The relationship between VM expression (VM + : CD31-/PAS + ; VM-: CD31 + /PAS-) and NPC clinicopathological characteristics.

\begin{tabular}{|c|c|c|c|c|c|}
\hline & & Total & $\mathrm{VM}+$ & VM- & $p$-value \\
\hline & Cases (n) & 114 & 78 & 36 & 0.577 \\
\hline \multirow[t]{2}{*}{ Sex } & Man & 80 & 56 & 24 & \\
\hline & Female & 34 & 22 & 12 & \\
\hline \multirow[t]{2}{*}{ Age } & $<50$ & 73 & 54 & 19 & 0.457 \\
\hline & $\geqq 50$ & 41 & 24 & 17 & \\
\hline Foxq1 & $4.00(2.57-6.00)$ & $4.75(3.00-6.54)$ & $2.66(1.50-4.14)$ & $<0.001$ & \\
\hline EGFR & $6.61 \pm 0.24$ & $7.36 \pm 0.26$ & $4.95 \pm 0.40$ & $<0.001$ & \\
\hline \multirow[t]{4}{*}{ TNM stage } & I & 7 & 3 & 4 & 0.013 \\
\hline & $\|$ & 24 & 11 & 13 & \\
\hline & III & 38 & 29 & 9 & \\
\hline & IV & 45 & 35 & 10 & \\
\hline \multirow[t]{4}{*}{ T stage } & 1 & 20 & 10 & 10 & 0.127 \\
\hline & 2 & 30 & 19 & 11 & \\
\hline & 3 & 25 & 20 & 5 & \\
\hline & 4 & 39 & 29 & 10 & \\
\hline \multirow[t]{4}{*}{ N stage } & 0 & 16 & 5 & 11 & 0.004 \\
\hline & 1 & 34 & 24 & 10 & \\
\hline & 2 & 50 & 37 & 13 & \\
\hline & 3 & 14 & 12 & 2 & \\
\hline \multirow[t]{4}{*}{ Foxq1 } & - & 5 & 1 & 4 & $<0.001$ \\
\hline & + & 36 & 16 & 20 & \\
\hline & ++ & 44 & 35 & 9 & \\
\hline & +++ & 29 & 26 & 3 & \\
\hline \multirow[t]{4}{*}{ EGFR } & - & 3 & 1 & 2 & $<0.001$ \\
\hline & + & 18 & 5 & 13 & \\
\hline & ++ & 56 & 39 & 17 & \\
\hline & +++ & 37 & 33 & 4 & \\
\hline
\end{tabular}

to predict Foxq1-binding sites in the EGFR promoter. Multiple binding sites were predicted (Fig. 4F) ${ }^{32}$ and two most likely binding sites were selected and verified by the luciferase reporter assay. First, we cloned the full-length (FL) EGFR promoter $(1 \mathrm{~kb})$ or the mutant EGFR promoter into the luciferase reporter plasmids. The mutant EGFR promoters contained three degenerate Foxq1-binding elements (TBEs), such as ATAGTTTAAA (m1), AATTAAACATTA $(\mathrm{m} 2)$ and both of them $(\mathrm{m} 3)$, respectively, (Fig. 4G). Then, the luciferase reporter plasmids were cotransfected with Foxq1 or control plasmids into 293T cells, and the fluorescence signal was detected after $36 \mathrm{~h}$. The result showed an increased transactivation of EGFR in the Foxq1 group compared with the control group. Furthermore, mutant $\mathrm{m} 1$ or $\mathrm{m} 2$ reduced the EFGR transactivation, while mutant $\mathrm{m} 3$ had no significant binding activity with Foxq1 (Fig. 4H). These results indicated that $\mathrm{m} 1$ and $\mathrm{m} 2$ sites are the main Foxq1binding sites in the EGFR promoter region. Next, we performed a chromatin immunoprecipitation (CHIP) assay to substantiate the interaction between Foxq1 and EGFR. CHIP-PCR showed that the anti-Foxq1 antibody successfully pulled down the predicted EGFR binding sequence (Fig. 4I). qRT-PCR analysis of the pulled down chromatins by Foxq1 and IgG antibodies implicated an enrichment of Foxq1 occupancies at the EGFR promoter region in the precipitated material with the Foxq1-specific antibody, but not with the IgG antibodies (Fig. 4J). These 


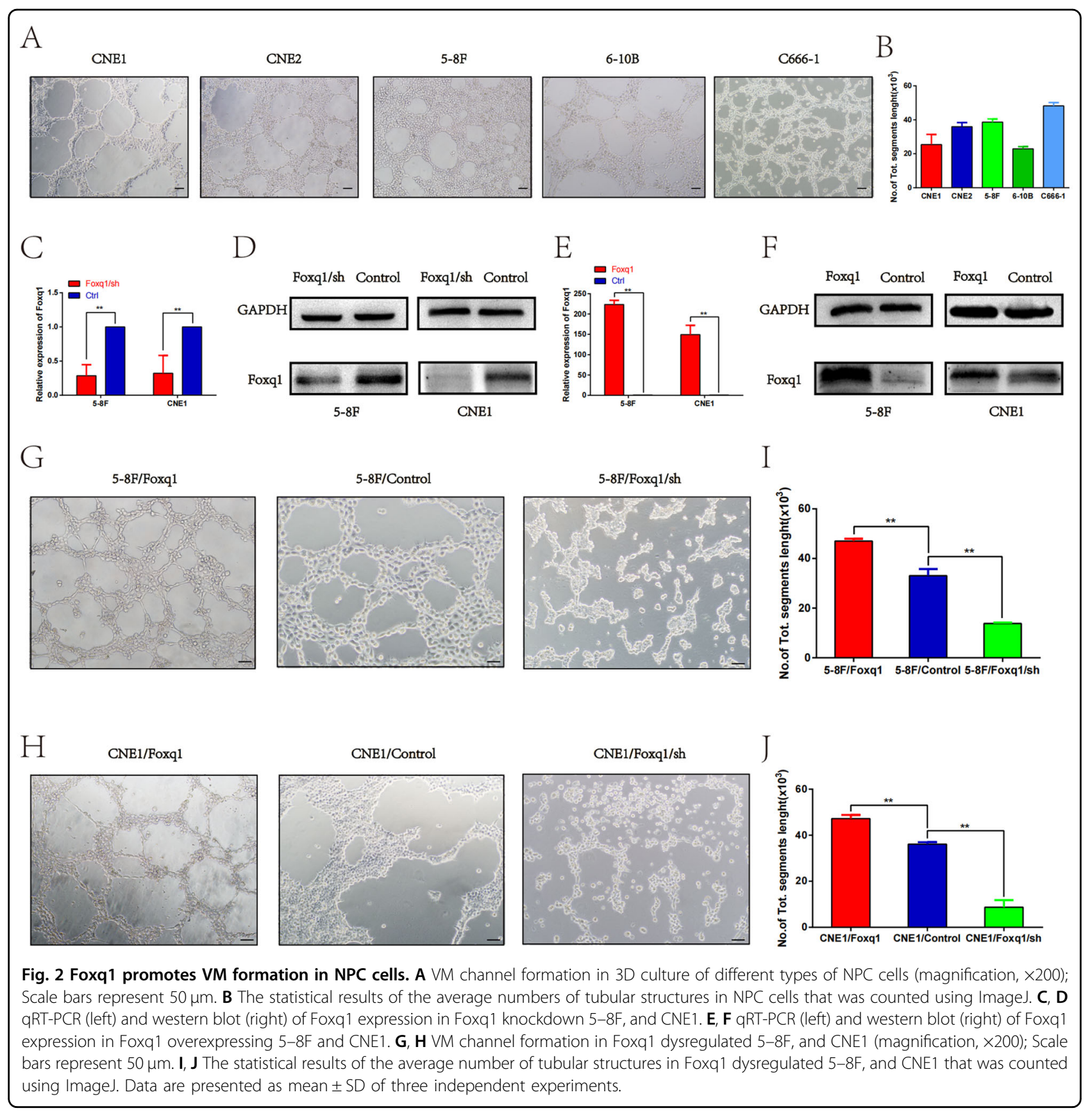

results support the possibility that Foxq1 directly regulates EGFR transcription. In conclusion, Foxq1 promotes EGFR transcription through its binding to the EGFR promoter $\mathrm{m} 1$ and $\mathrm{m} 2$ sites.

\section{Foxq1 promotes VM formation, and NPC growth and metastasis in vivo}

We investigated in vivo the relationship between Foxq1, NPC growth and VM formation using a subcutaneous $\mathrm{BALB} / \mathrm{c}$ nude mice xenograft tumor model ( $n=6 /$ group). Control and Foxq1stably overexpressing 5-8F cells were subcutaneously injected into BALB/c-nude mice, respectively. Through plotting the tumor growth curves, we found that tumors' volume in the Foxq1-overexpressing group was significantly higher than that in the control group (Fig. 5A, B). Meanwhile, the tumors' weight in the Foxq1overexpressing group was significantly higher than that in the control group (Fig. 5C). The histologic analysis of the tumors indicated that the number of the VM events and the expression of EGFR, were significantly increased in the Foxq1-overexpressing group compared with those in the control group (Fig. 5D-G). Consistent with the Foxq1 


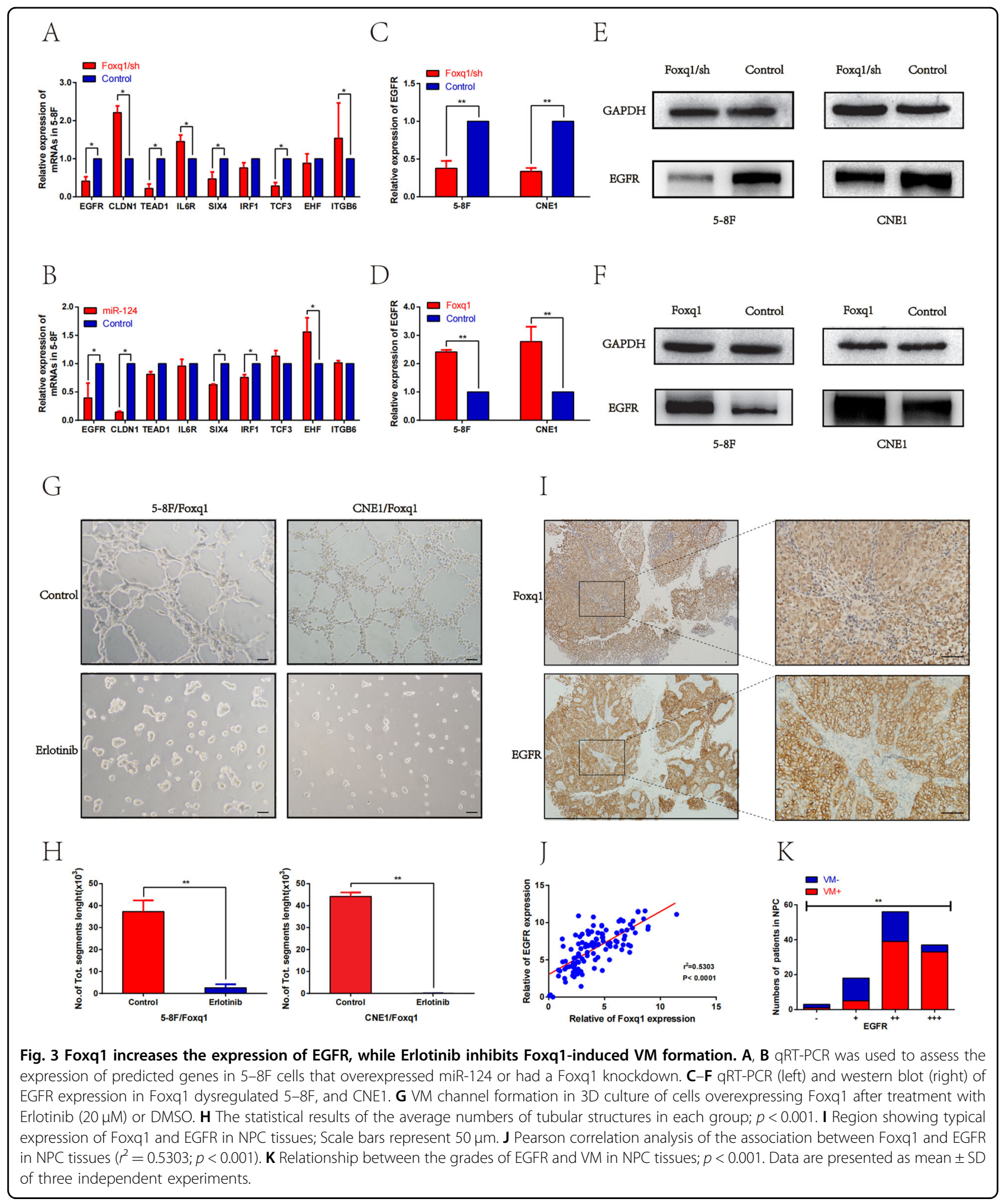

tumor-promoting effects, qRT-PCR and western blot showed that the expression of related genes in the tumors were also dramatically increased in Foxq1-overexpressing group compared to those in the control group (Fig. 5J, K).
These results show that Foxq1 promotes VM formation and NPC growth by increasing EGFR expression in vivo.

Moreover, an in vivo lung metastasis model was chosen to further verify Foxq1 role in NPC metastasis. The 


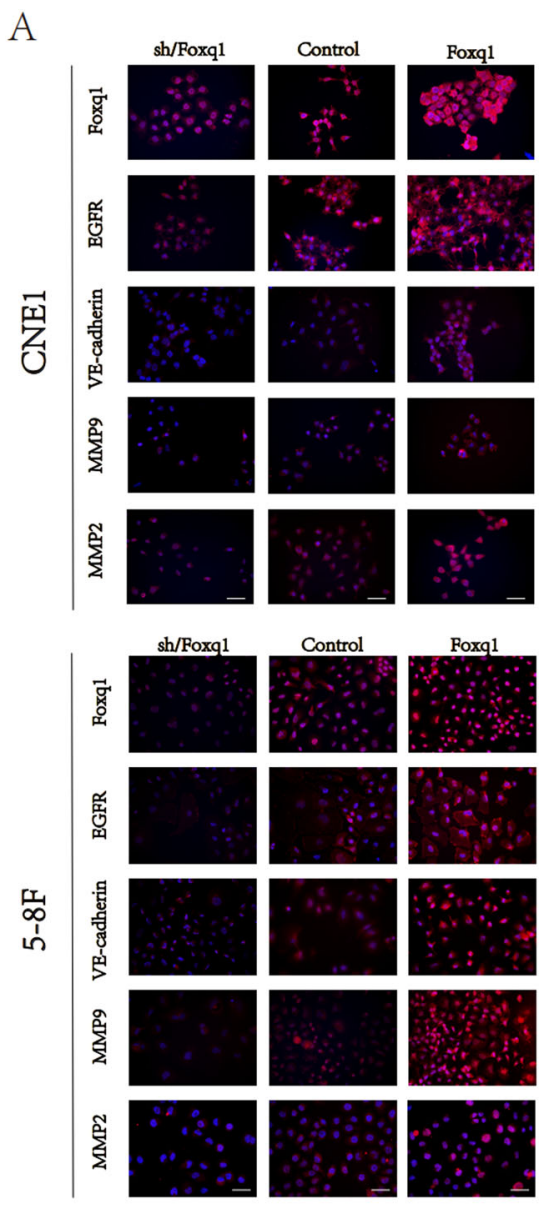

B
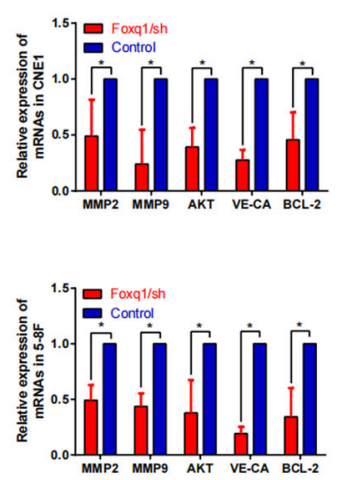

C

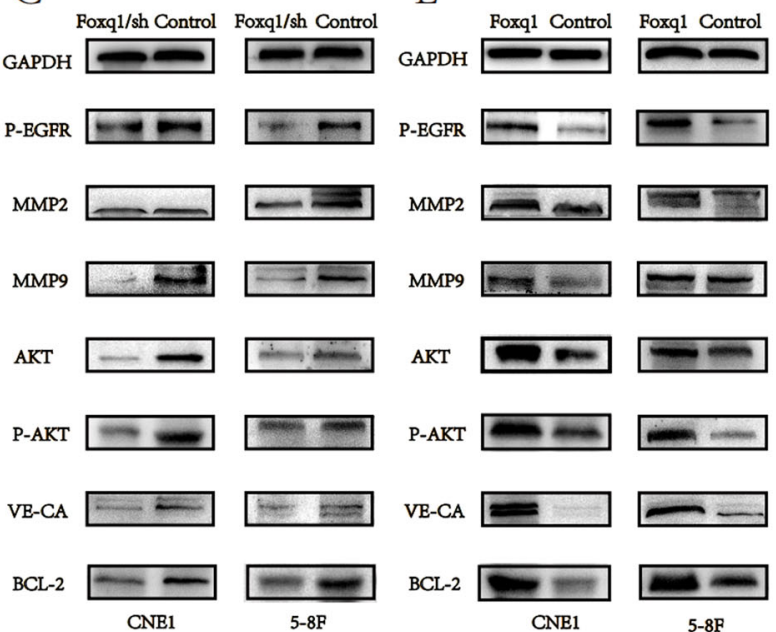

G

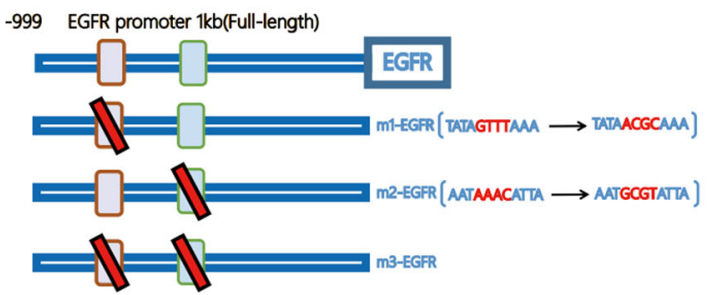

D
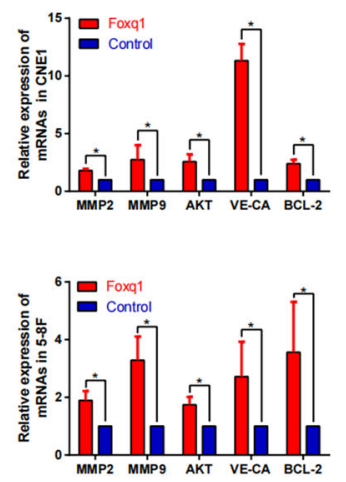

$\mathrm{E}$
$\mathrm{H}$

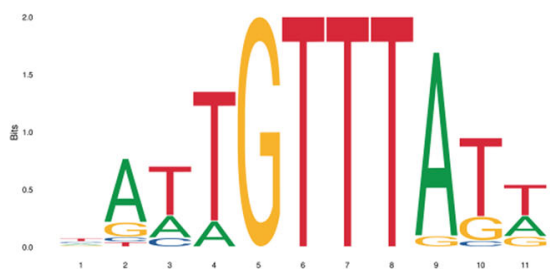

I

F
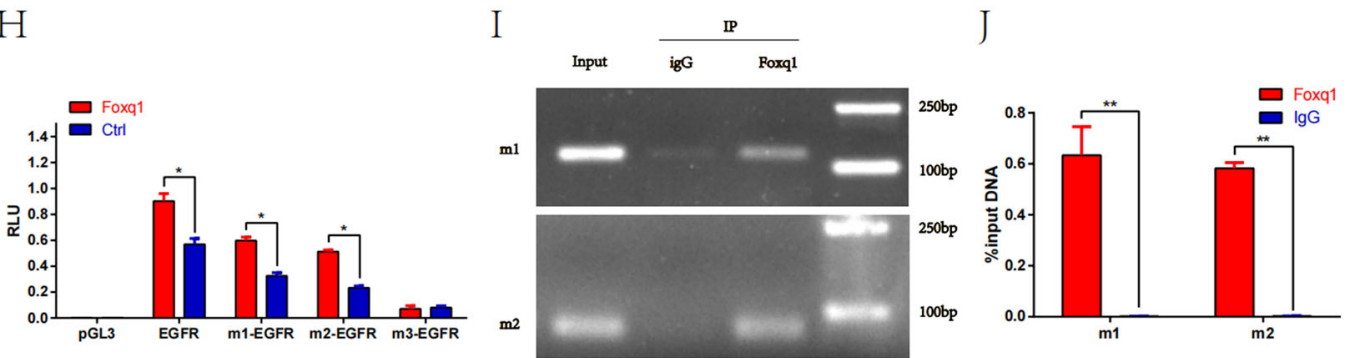

Fig. 4 Foxq1 promotes the EGFR signaling pathway and VM-related genes through a direct binding to the EGFR promoter. A Immunofluorescence staining of Foxq1,EGFR,VE-cadherin,MMP2, and MMP9 in Foxq1 dysregulated 5-8F, and CNE1; Scale bars represent 50 m. qRTPCR (above) and western blot (down) were used to monitor the expression of EGFR signaling pathway and VM-related genes in 5-8F, and CNE1 after Foxq1 downregulation $(\mathbf{B}, \mathbf{C})$ or overexpression $(\mathbf{D}, \mathbf{E})$. $\mathbf{F}$ The binding motif of Foxq1 from the JASPAR database. $\mathbf{G}$ A diagram showing the relationship of full-length (FL) and mutant EGFR promoters. $\mathbf{H} 293$ T cells were transfected with Foxq1 overexpressing or control vector. The luciferase reporter gene vectors carrying FL or EGFR promoter mutant were co-transfected respectively for $36 \mathrm{~h}$. Then, the dual-luciferase activity was detected. I The CHIP-PCR assay was used to assess the binding of Foxq1 to the EGFR promoter region. J Anti-Foxq1-pulled down chromatins were analyzed by qRTPCR. Data are presented as mean \pm SD of three independent experiments. 


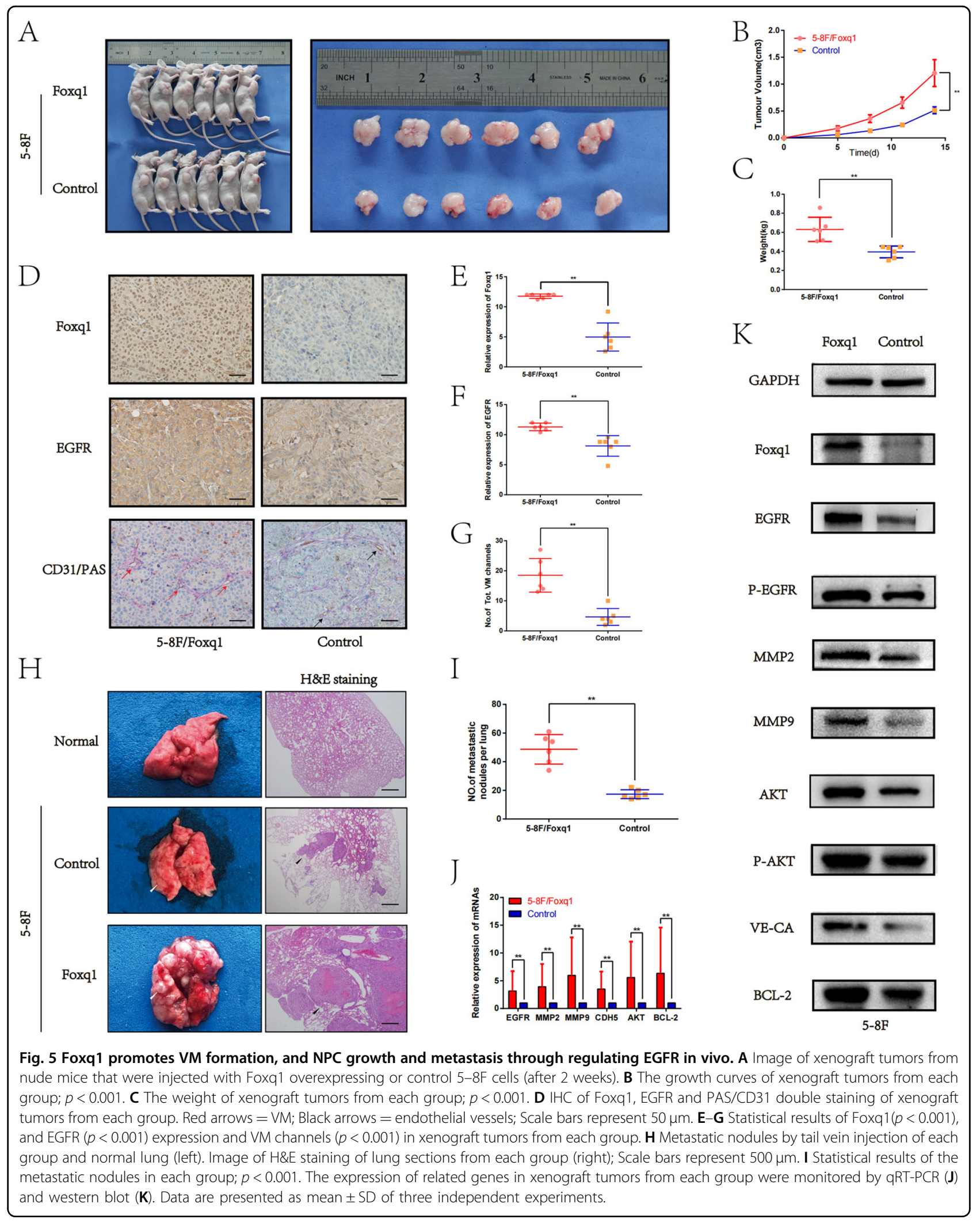


control and Foxq1 overexpressing 5-8F cells were inoculated into BALB/c-nude mice through tail vein, respectively. Five weeks later, their metastatic capacity was assessed through counting metastatic nodules in the lung. The results showed that the mice that were injected with Foxq1 overexpressing $5-8$ F cells formed significantly more metastatic nodules compared with the control mice (Fig. 5H, I). These results demonstrate that Foxq1 promotes NPC metastasis in vivo.

\section{In vivo, Erlotinib and Nimotuzumab inhibit Foxq1-induced} VM formation, and NPC growth and metastasis

To further investigate the effect of EGFR on Foxq1induced VM, and NPC growth and metastasis in vivo, we injected the Foxq1 overexpressing 5-8F cells into BALB/ c-nude mice subcutaneously or through the tail vein, then treated them with Erlotinib, Nimotuzumab or physiological saline, respectively. As expected, the tumors that were treated with Erlotinib or Nimotuzumab grew slower than those in the control group (Fig. 6B), while the volume and weight were also smaller than those in the control group (Fig. 6A and Fig. S2). Consistent with these results, IHC of the tumors using a PAS/CD31 double staining indicated that the number of $\mathrm{VM}$ events was significantly decreased in the Erlotinib or Nimotuzumab group compared with that of the control group (Fig. 6C, D). These results were further confirmed by investigating the expression of related genes that were assessed by qRTPCR and western blot (Fig. 6G, H). In the lung metastasis model, the mice that were treated with Erlotinib and Nimotuzumab formed significantly less metastatic nodules compared that to the mice that were treated with physiological saline (Fig. 6E, F). In summary, these results demonstrate that, as the EGFR inhibitors, Erlotinib and Nimotuzumab, prevent Foxq1 from promoting VM formation, and NPC growth and metastasis.

\section{Combination with anti-EGFR and anti-VEGF drugs resulted in improved antitumor efficacy}

Above results have revealed that anti-EGFR therapy is an effective strategy in inhibiting Foxq1-induced VM formation. While anti-VEGF therapy, which was used to prevent endothelium-dependent vessels (EDV), has been reported to have no inhibitory effect on $\mathrm{VM}^{13}$. Hence, it is worth to explore the combined effect of anti-EGFR therapy and anti-VEGF therapy. One week after Foxq1 overexpressing 5-8F cells were injected into BALB/cnude mice subcutaneously, we treated them with Sunitinib, combination of Sunitinib and Erlotinib, or combination of Sunitinib and Nimotuzumab, respectively (Fig. S3). Although Sunitinib showed effective inhibition of tumors, the combination of anti-EGFR and Sunitinib groups grew slower than those in the group treated Sunitinib alone (Fig. 6I). The volume and weight (Figs. S4 and S5) of the combination treatment groups were also smaller than those in the control group or treated Sunitinib alone. Through CD31-PAS dual staining, we found that Sunitinib had strong inhibitory effect on angiogenesis, but no obviously effect on VM, While the combination of antiEGFR and Sunitinib showed a significantly negative effect on both EDV and VM (Fig. 6J-L). These results confirmed the synergistic effect of anti-VEGF and anti-EGFR drugs, providing a new therapeutic option for nasopharyngeal carcinoma.

\section{Mir-124 inhibits the expression of EGFR and VM formation, events that are rescued by Foxq1}

Our previous study confirmed that miR-124 could target Foxq1 to inhibit NPC growth and metastasis. In this study, we verified the VM formation capacity of the miR124 overexpressing $5-8 \mathrm{~F}$ cells and found that it was significantly reduced compared with the control group in the 3D cultures assay (Fig. 7A, C). To investigate the role of Foxq1 and EGFR in miR-124-induced VM inhibition, we conducted rescue experiments by overexpressing Foxq1 in 5-8f cells that also overexpressed miR-124. The results showed that Foxq1 could reverse the inhibitory effect of miR-124 on VM formation, and that was also inhibited by Erlotinib (Fig. 7B, D). Furthermore, we found that miR124 overexpression significantly reduces the expressions of Foxq1, and EGFR signaling pathway and VM-related genes as demonstrated using qRT-PCR, western blot and Immunofluorescence staining (Fig. 7E-G). These data strongly support our hypothesis that the miR-124/Foxq1 axis inhibits VM formation through the EGFR signaling pathway (Fig. 8).

\section{Discussion}

During tumorigenesis, oxygen and nutrients that are provided by blood, are indispensable. When reaching a diameter of $\geq 2 \mathrm{~mm}$, the tumor needs a new blood-supply to meet its basic metabolic requirements ${ }^{33}$. Vasculogenic mimicry is surrounded by highly invasive tumor cells that are PAS-positive and CD31-negative staining highly invasive tumor cells, and provides sufficient blood-supply for tumor tissues ${ }^{8}$. Owing to this unique structure, tumor cells are in direct contact with the blood, which greatly increases the risk of distant blood metastasis ${ }^{34}$. Hence, VM frequently correlates with an advanced tumor grade, invasion, metastasis and poor prognosis ${ }^{35}$.

Our study confirmed the presence of vasculogenic mimicry in nasopharyngeal carcinoma tissues using a CD31-PAS dual staining. VM was significantly related to clinical stage, and the Kaplan-Meier analysis indicated that patients who are VM positive had a shorter survival time. Furthermore, vasculogenic mimicry in nasopharyngeal carcinoma was further detected in vitro and in vivo. These results indicated that VM is a poor prognostic indicator in nasopharyngeal 


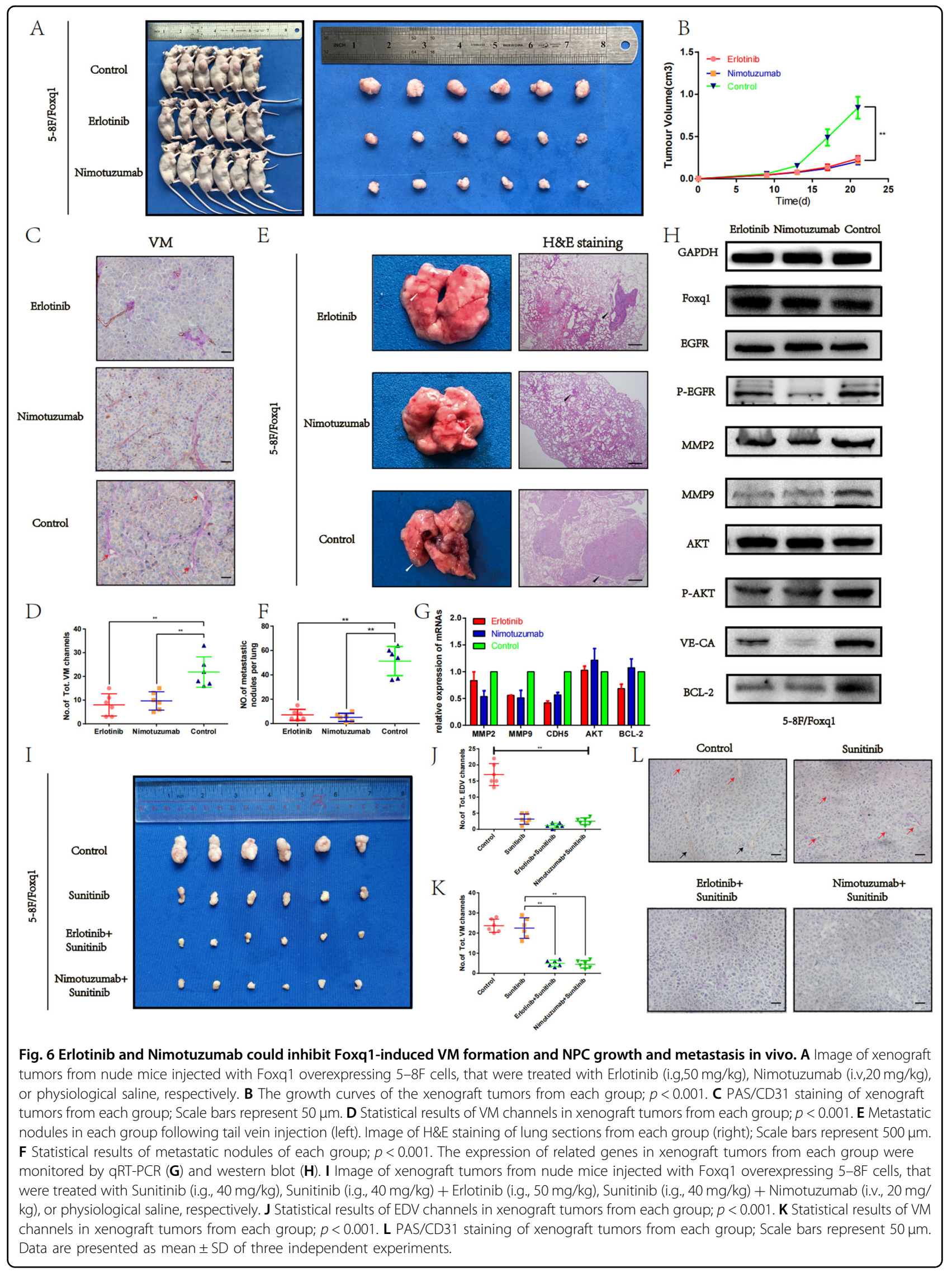




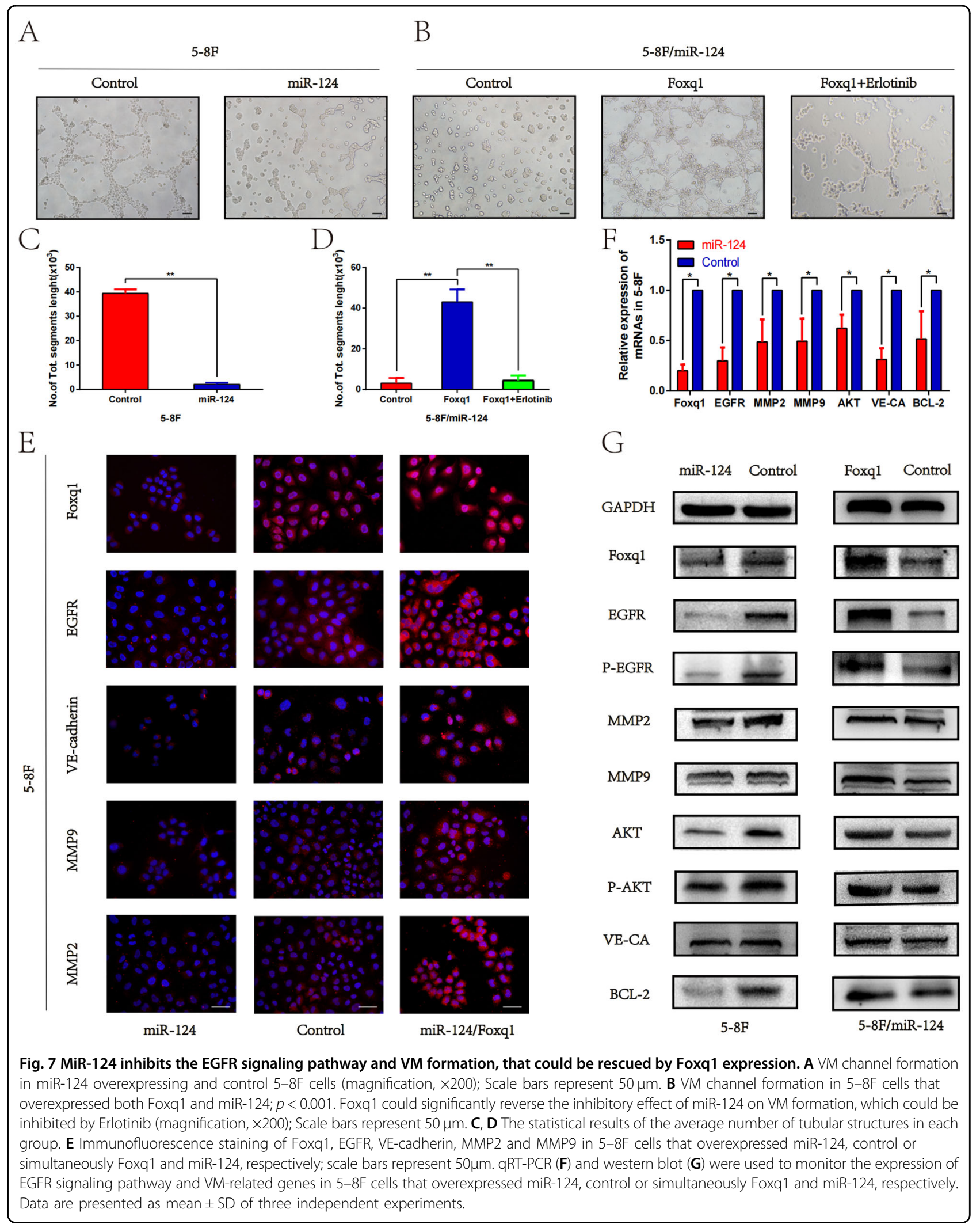




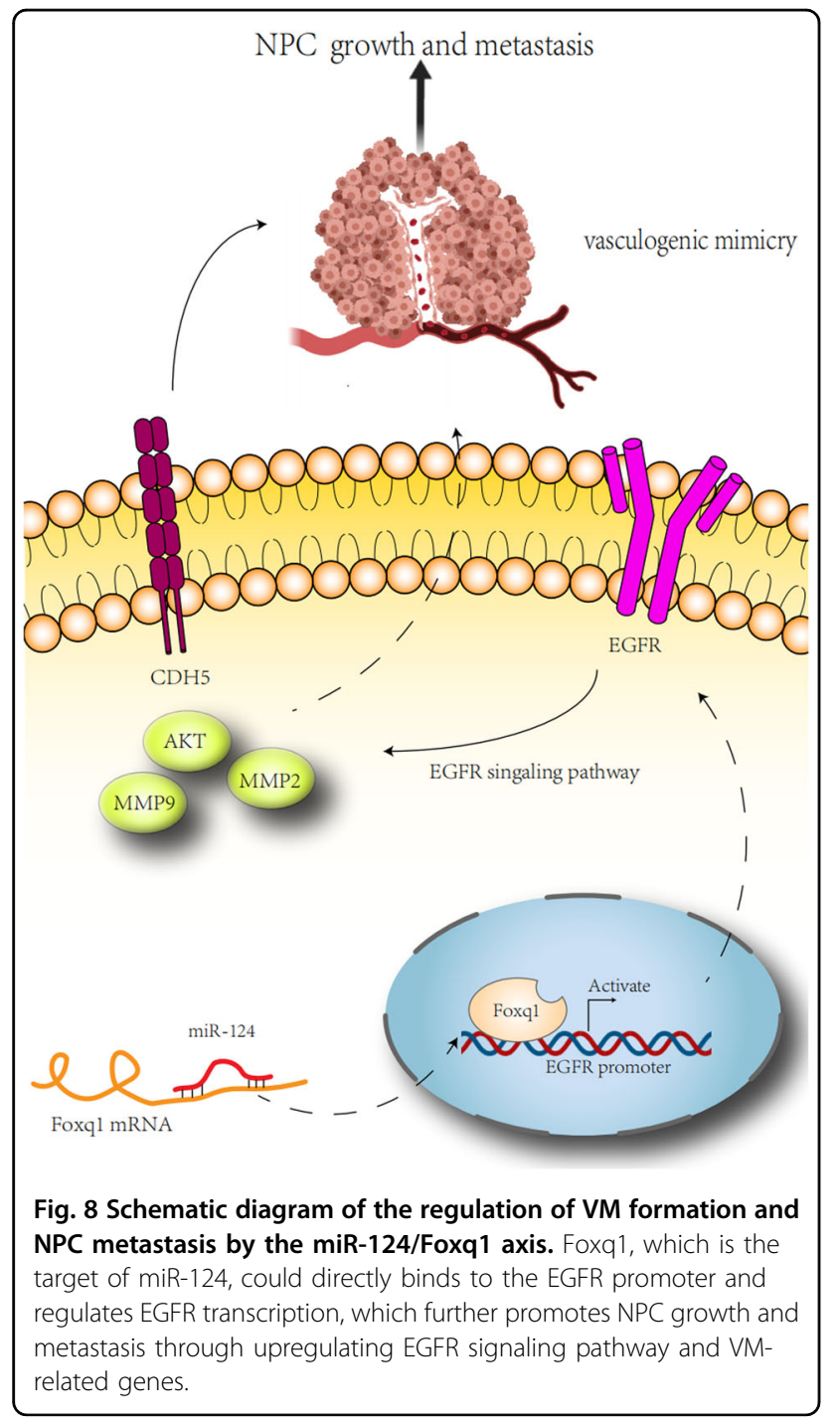

carcinoma patients and that it plays an important role in its development.

Currently, the mechanism of VM regulation is controversial. Multiple molecular mechanisms, especially vascular endothelial (VE)-cadherin, erythropoietinproducing hepatocellular receptor A2 (EphA2), phosphatidyl inositol 3-kinase (PI3K), matrix metalloproteinases (MMPs), vascular endothelial growth factor receptor (VEGFR1), and hypoxia inducible factor (HIF)$1 \alpha$, have been reported to participate in VM formation that is associated with tumor migration and invasion ${ }^{36,37}$. In addition, hypoxia, cancer stem cells (CSCs) and epithelial-mesenchymal transition (EMT) are regarded as significant factors in VM formation and tumor metastasis $^{38}$. However, the exact mechanism of VM formation is still unclear and requires further studies.

In this study, we found that Foxq1 promotes EGFR expression at mRNA and protein levels, and that EGFR inhibitor drugs could inhibit Foxq1-induced VM formation. In vivo, Foxq1 promoted the growth and metastasis of nasopharyngeal carcinoma, which could be prevented by EGFR inhibitors. Meanwhile, the EGFR signaling pathway and VM, were significantly inhibited in the group that was treated with EGFR inhibitor drugs. Furthermore, Luciferase reporter gene and CHIP assays showed that Foxq1 directly binds to the EGFR promoter region and regulates its transcription. The above results indicate that Foxq1 is regulated by miR-124, which promoted NPC metastasis by inducing VM via the EGFR signaling pathway.

EGFR could activate a series of downstream signaling pathways to exert corresponding biological effects, such as cell proliferation, apoptosis and metastatic spread ${ }^{39}$. Meanwhile, EGFR has been proved to be overexpressed and acted as a poor prognostic factor in a variety of tumors, including head and neck, breast, lung, and colorectal cancers ${ }^{40}$; hence, EGFR drugs are widely used in cancer therapy. In nasopharyngeal carcinoma, EGFR expression rate was as high as $90 \%$, and it was closely related to chemoresistance, radioresistance and poor prognosis ${ }^{41}$. Owing to its critical role, anti-EGFR therapy is considered to be an effective target in NPC treatment ${ }^{42}$, and therefore, it is necessary to clarify the specific mechanism of anti-EGFR targeted drugs in the treatment of nasopharyngeal carcinoma.

The mechanism of EGFR overactivation during tumor pathogenesis is mainly associated with the overexpression of EGFR or its ligands ${ }^{43}$. For example, the long noncoding RNA, EGFR-AS1, was shown to directly binds EGFR mRNA, which promotes cell growth and metastasis through upregulating EGFR expression in renal cancer ${ }^{44}$. While a recent study has shown that CD317 is associated with lipid rafts, and activates EGFR in hepatocellular carcinoma (HCC) cells by regulating its localization on the plasma membrane ${ }^{45}$. In this study, we demonstrated that Foxq1 directly binds to the EGFR promoter region and regulates its transcription. Furthermore, VM was regulated by the miR-124-Foxq1-EGFR axis in nasopharyngeal carcinoma. The above results illustrated that the inhibition of VM formation by the miR-124-Foxq1EGFR axis might be the new mechanism of anti-EGFR drugs in the treatment of nasopharyngeal carcinoma.

Our results also indicated that the presence of VM may be a possible cause of anti-VEGF drugs' failure in achieving satisfactory results in nasopharyngeal carcinoma treatment. Researchers have confirmed that effective therapeutic strategies should simultaneously inhibit VM and $\mathrm{EDV}^{46}$. Meanwhile, encouraging results has also been observed in clinical trials using a combination of VEGF and EGFR blockade $^{47}$. For example, in non-small-cell lung cancer, the combination of Bevacizumab and Erlotinib has been confirmed to improve progression-free survival compared with 
erlotinib alone in a phase 3 trial $^{48}$. In addition, the combination of Bevacizumab and Erlotinib has been shown to be in the treatment of metastatic renal cell carcinoma and special types of advanced breast cancer ${ }^{49,50}$. A randomized phase II trial also showed that the combination of bevacizumab and cetuximab might be a sensible treatment strategy in metastatic colorectal cancer ${ }^{51}$. Evidence from these trials supported the feasibility and efficacy of developing a combined VEGF and EGFR inhibition therapy for patients with solid tumors. Based on our research about the synergistic effect of anti-VEGF and anti-EGFR drugs, we consider that the combination of anti-EGFR and anti-VEGF therapy is an effective therapeutic strategy for advanced or metastatic nasopharyngeal carcinoma.

\section{Conclusions}

In conclusion, we provide evidence that the miR-124Foxq1-EGFR axis contributes to NPC growth and metastasis through regulating VM formation. These results provide new targets and options for nasopharyngeal carcinoma anti-VM therapy. Meanwhile, we demonstrate that VM, as a poor prognostic factor for nasopharyngeal carcinoma, can be inhibited by Erlotinib and Nimotuzumab. These suggest that anti-EGFR therapy is an effective strategy in inhibiting $\mathrm{VM}$ formation and in preventing metastasis of nasopharyngeal carcinoma. Finally, the combination of anti-EGFR and anti-VEGF therapy shows better tumor inhibition than single anti-VEGF therapy, which is beneficial to optimize the clinical treatment of NPC.

\section{Acknowledgements}

This research was supported the National Natural Science Foundation of China (81702696 and 81472535), Natural Science Foundation of Guangdong Province of China (2017A030310040) and Medical Scientific Research Foundation of Guangdong Province of China(A2020078).

\section{Author contributions}

Y.F.L. executed all experiments. J.W. and F.W. assisted in bioinformatic analysis. X.M.M. and X.X.Y. were responsible for clinical sample collection. X.L. and J.L. assisted in statistical analysis. X.H.P. and X.P.L. made critical modifications to the manuscript. Y.F.L. and X.H.P. designed and supervised research and wrote the manuscript. All authors read and approved the final manuscript.

\section{Funding}

This study was supported by grants from the National Natural Science Foundation of China (81702696 and 81472535), Natural Science Foundation of Guangdong Province of China (2017A030310040) and Medical Scientific Research Foundation of Guangdong Province of China (A2020078).

\section{Data availability}

The datasets during the current study available from the corresponding author on reasonable request.

\section{Conflict of interest}

The authors declare no competing interests.

\section{Ethics approval}

All clinical studies were approved by the Ethics Committee of Southern Medical University. All animal experiments were performed according to guidelines approved by the Institutional Animal Care And Use Committee of Southern Medical University.
Informed consent

Informed written consent was obtained from each patient.

\section{Publisher's note}

Springer Nature remains neutral with regard to jurisdictional claims in published maps and institutional affiliations.

Supplementary information The online version contains supplementary material available at https://doi.org/10.1038/s41419-021-03674-z.

Received: 9 January 2021 Accepted: 25 March 2021

Published online: 19 April 2021

\section{References}

1. Zou, X. et al. Establishment and validation of M1 stage subdivisions for de novo metastatic nasopharyngeal carcinoma to better predict prognosis and guide treatment. Eur. J. Cancer (Oxf., Engl.: 1990) 77, 117-126 (2017).

2. You, R. et al. Efficacy and safety of locoregional radiotherapy with chemotherapy vs chemotherapy alone in de novo metastatic nasopharyngeal carcinoma: a multicenter phase 3 randomized clinical trial. JAMA Oncol. 6, 1345-1352 (2020).

3. Sun, X. et al. The association between the development of radiation therapy, image technology, and chemotherapy, and the survival of patients with nasopharyngeal carcinoma: a cohort study from 1990 to 2012. Int. J. Radiat. Oncol. Biol. Phys. 105, 581-590 (2019).

4. Hanahan, D. \& Weinberg, R. A. Hallmarks of cancer: the next generation. Cell 144, 646-674 (2011).

5. Hui, E. P. et al. Hemorrhagic complications in a phase II study of sunitinib in patients of nasopharyngeal carcinoma who has previously received high-dose radiation. Ann. Oncol.: Off. J. Eur. Soc. Med. Oncol. 22, 1280-1287 (2011).

6. Lee, N. Y. et al. Addition of bevacizumab to standard chemoradiation for locoregionally advanced nasopharyngeal carcinoma (RTOG 0615): a phase 2 multi-institutional trial. Lancet Oncol. 13, 172-180 (2012).

7. Maniotis, A. et al. Vascular channel formation by human melanoma cells in vivo and in vitro: vasculogenic mimicry. Am. J. Pathol. 155, 739-752 (1999).

8. Leslie, M. Tumors have found a bloody new way to grow and spread. Science https://doi.org/10.1126/science.aag0605 (2016).

9. Seftor, R. et al. Cooperative interactions of laminin 5 gamma2 chain, matrix metalloproteinase-2, and membrane type-1-matrix/metalloproteinase are required for mimicry of embryonic vasculogenesis by aggressive melanoma. Cancer Res. 61, 6322-6327 (2001).

10. Pinto, M., Sotomayor, P., Carrasco-Avino, G., Corvalan, A. \& Owen, G. Escaping antiangiogenic therapy: strategies employed by cancer cells. Int. J. Mol. Sci. 17, 9 (2016).

11. Sun, $\mathrm{H}$. et al. Anti-angiogenic treatment promotes triple-negative breast cancer invasion via vasculogenic mimicry. Cancer Biol. Ther. 18, 205-213 (2017).

12. Hu, Y. L., Jahangiri, A., De Lay, M. \& Aghi, M. K. Hypoxia-induced tumor cell autophagy mediates resistance to anti-angiogenic therapy. Autophagy $\mathbf{8}$, 979-981 (2012).

13. $\mathrm{Xu}$, Y. et al. Short-term anti-vascular endothelial growth factor treatment elicits vasculogenic mimicry formation of tumors to accelerate metastasis. J. Exp. Clin. Cancer Res. 31, 16 (2012).

14. Hernández de la Cruz, O. et al. Regulation networks driving vasculogenic mimicry in solid tumors. Front. Oncol. 9, 1419 (2019).

15. Yang, Z. et al. Androgen receptor suppresses prostate cancer metastasis but promotes bladder cancer metastasis via differentially altering miRNA525-5p/ SLPI-mediated vasculogenic mimicry formation. Cancer Lett. 473, 118-129 (2020).

16. $\mathrm{Xu}$, S. et al. EBV-LMP1 is involved in vasculogenic mimicry formation via VEGFANEGFR1 signaling in nasopharyngeal carcinoma. Oncol. Rep. 40, 377-384 (2018).

17. Xiang, T. et al. Vasculogenic mimicry formation in EBV-associated epithelial malignancies. Nat. Commun. 9, 5009 (2018).

18. Peng, X. H. et al. MiR-124 suppresses tumor growth and metastasis by targeting Foxq1 in nasopharyngeal carcinoma. Mol. Cancer 13, 186 (2014). 
19. Li, Y. et al. Forkhead box Q1: A key player in the pathogenesis of tumors (Review). Int. J. Oncol. 49, 51-58 (2016).

20. Zhang, J. et al. FOXQ1 promotes gastric cancer metastasis through upregulation of Snail. Oncol. Rep. 35, 3607-3613 (2016).

21. Jiao, D. et al. LnCRNA MALAT1 promotes tumor growth and metastasis by targeting miR-124/foxq1 in bladder transitional cell carcinoma (BTCC). Am. J. Cancer Res. 8, 748-760 (2018).

22. Zhu, Z. et al. Short hairpin RNA targeting FOXQ1 inhibits invasion and metastasis via the reversal of epithelial-mesenchymal transition in bladder cancer. Int. J. Oncol. 42, 1271-1278 (2013).

23. Wei, $\mathrm{C}$. et al. Crosstalk between cancer cells and tumor associated macrophages is required for mesenchymal circulating tumor cell-mediated colorectal cancer metastasis. Mol. Cancer 18, 64 (2019).

24. Xiang, X. et al. MiR-1271 Inhibits Cell Proliferation, Invasion and EMT in Gastric Cancer by Targeting FOXQ1. Cell. Physiol. Biochem.: Int. J. Exp. Cell. Physiol., Biochem., Pharmacol. 36, 1382-1394 (2015).

25. Sun, T. et al. Expression and functional significance of Twist1 in hepatocellular carcinoma: its role in vasculogenic mimicry. Hepatology 51, 545-556 (2010).

26. Lin, $\mathrm{X}$. et al. C-myc overexpression drives melanoma metastasis by promoting vasculogenic mimicry via c-myc/snail/Bax signaling. J. Mol. Med. (Berl., Ger.) 95, 53-67 (2017).

27. Liu, Z. et al. Zinc finger E-box binding homeobox 1 promotes vasculogenic mimicry in colorectal cancer through induction of epithelial-to-mesenchymal transition. Cancer Sci. 103, 813-820 (2012).

28. Ma, B. et al. A phase II study of patients with metastatic or locoregionally recurrent nasopharyngeal carcinoma and evaluation of plasma Epstein-Barr virus DNA as a biomarker of efficacy. Cancer Chemother. Pharmacol. 62, 59-64 (2008).

29. You, R. et al. Cetuximab or nimotuzumab plus intensity-modulated radiotherapy versus cisplatin plus intensity-modulated radiotherapy for stage II-IVb nasopharyngeal carcinoma. Int. J. Cancer 141, 1265-1276 (2017).

30. Gao, Y. et al. Long non-coding RNA HOXA-AS2 regulates malignant glioma behaviors and vasculogenic mimicry formation via the MiR-373/EGFR axis. Cell. Physiol. Biochem.: Int. J. Exp. Cell. Physiol., Biochem., Pharmacol. 45, 131-147 (2018).

31. Camorani, S. et al. Aptamer-mediated impairment of EGFR-integrin av $\beta 3$ complex inhibits vasculogenic mimicry and growth of triple-negative breast cancers. Sci. Rep. 7, 46659 (2017).

32. Khan, A. et al. JASPAR 2018: update of the open-access database of transcription factor binding profiles and its web framework. Nucleic Acids Res. 46, D260-D266 (2018)

33. Jakóbisiak, M., Lasek, W. \& Gołab, J. Natural mechanisms protecting against cancer. Immunol. Lett. 90, 103-122 (2003).

34. Zhang, X., Zhang, J., Zhou, H., Fan, G. \& Li, Q. Molecular mechanisms and anticancer therapeutic strategies in vasculogenic mimicry. J. Cancer 10, 6327-6340 (2019).
35. Yang, J. et al. Tumor vasculogenic mimicry predicts poor prognosis in cancer patients: a meta-analysis. Angiogenesis 19, 191-200 (2016).

36. Delgado-Bellido, D., Serrano-Saenz, S., Fernández-Cortés, M. \& Oliver, F. Vasculogenic mimicry signaling revisited: focus on non-vascular VE-cadherin. Mol. Cancer 16, 65 (2017).

37. Wang, M. et al. HIF-1a promoted vasculogenic mimicry formation in hepatocellular carcinoma through LOXL2 up-regulation in hypoxic tumor microenvironment. J. Exp. Clin. Cancer Res. 36, 60 (2017).

38. Luo, Q. et al. Vasculogenic mimicry in carcinogenesis and clinical applications. J. Hematol. Oncol. 13, 19 (2020).

39. Chen, Z. et al. EGFR family and Src family kinase interactions: mechanics matters? Curr. Opin. Cell Biol. 51, 97-102 (2018).

40. Nicholson, R., Gee, J. \& Harper, M. EGFR and cancer prognosis. Eur. J. Cancer 2001, S9-S15 (2001).

41. Tsang, J., Lee, V. \& Kwong, D. Novel therapy for nasopharyngeal carcinoma-where are we. Oral. Oncol. 50, 798-801 (2014).

42. Almobarak, A., Jebreel, A. \& Abu-Zaid, A. Molecular targeted therapy in the management of recurrent and metastatic nasopharyngeal carcinoma: a comprehensive literature review. Cureus 11, e4210 (2019).

43. Ayati, A. et al. A review on progression of epidermal growth factor receptor (EGFR) inhibitors as an efficient approach in cancer targeted therapy. Bioorg. Chem. 99, 103811 (2020).

44. Wang, A. et al. Long noncoding RNA EGFR-AS1 promotes cell growth and metastasis via affecting HuR mediated mRNA stability of EGFR in renal cancer. Cell Death Dis. 10, 154 (2019).

45. Zhang, G. et al. CD317 activates EGFR by regulating its association with lipid rafts. Cancer Res. 79, 2220-2231 (2019).

46. Wang, Y. et al. CRGD-functionalized nanoparticles for combination therapy of anti-endothelium dependent vessels and anti-vasculogenic mimicry to inhibit the proliferation of ovarian cancer. Acta Biomaterialia 94, 495-504 (2019).

47. Tabernero, J. The role of VEGF and EGFR inhibition: implications for combining anti-VEGF and anti-EGFR agents. Mol. Cancer Res.: MCR 5, 203-220 (2007).

48. Saito, $H$. et al. Erlotinib plus bevacizumab versus erlotinib alone in patients with EGFR-positive advanced non-squamous non-small-cell lung cancer (NEJ026): interim analysis of an open-label, randomised, multicentre, phase 3 trial. Lancet Oncol. 20, 625-635 (2019).

49. Hainsworth, J. et al. Treatment of metastatic renal cell carcinoma with a combination of bevacizumab and erlotinib. J. Clin. Oncol.: Off. J. Am. Soc. Clin. Oncol. 23, 7889-7896 (2005).

50. Montagna, E. et al. Metronomic chemotherapy combined with bevacizumab and erlotinib in patients with metastatic HER2-negative breast cancer: clinical and biological activity. Clin. Breast Cancer 12, 207-214 (2012).

51. Parisi, A. et al. Evaluation of second-line anti-VEGF after first-line Anti-EGFR based therapy in RAS wild-type metastatic colorectal cancer: the Multicenter "SLAVE" Study. Cancers 12, 1259 (2020). 